Health and Medicine | Professor David Wishart

\title{
Using metabolomics and artificial intelligence to explore the universe of the dark metabolome
}

Professor David Wishart,
University of Alberta, is a
member of Canada's national
metabolomics laboratory and
leads the Human Metabolome
Project. His work aims to identify
and catalogue metabolites in
human tissues, biofluids and
the surrounding environment.
He has developed a software
package called BioTransformer,
which is a chemical prediction
tool to explore the mysterious
dark metabolome. The program
combines artificial intelligence
with a knowledgebase to predict
and identify novel chemicals that
make up the dark metabolome.

to describe chemical

I reactions in the body, and the comprehensive, high-throughput study of metabolism is referred to as metabolomics. There are two main types of metabolism; catabolism is energy, and anabolism is the sythesis of comp and whis is the synthesis by cells of the body In addition, metabolism can refer to the activation, detoxification and elimination of foreig or unwanted substances.

In humans, metabolism is a complex process involving thousands of different enzymes which break down different foods, drugs and contaminants into

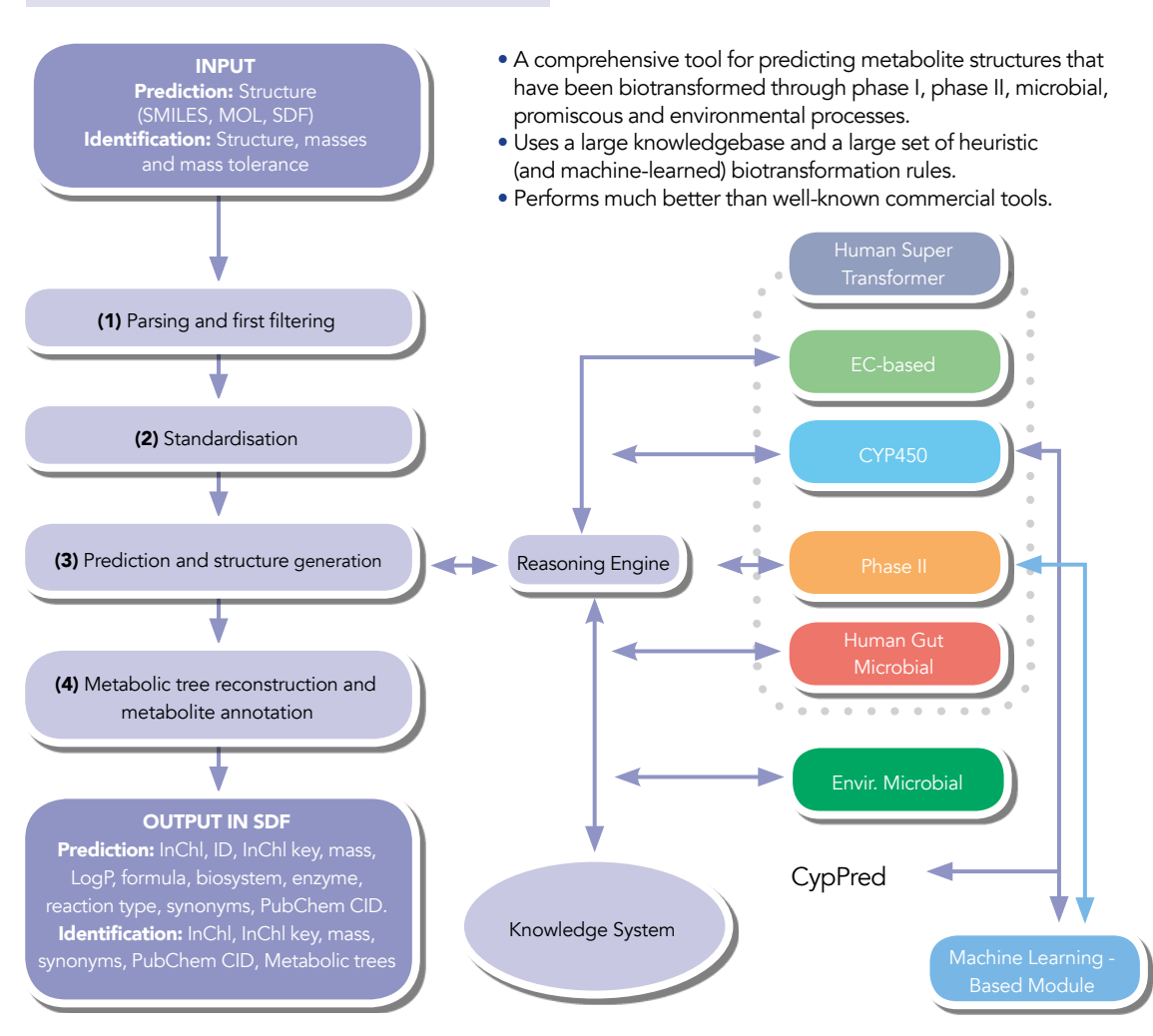

smaller chemical compounds which can be used, excreted or further transformed to other substances by specific enzymes in the body (biotransformation). These biotransformation enzymes are produced by different organs in the body including the liver, kidney, stomach and in the intestine (the microbiome)

The end result of this enzyme-driven process is hundreds of thousands of different chemicals (metabolites) circulating around the body. Metabolites can be beneficial or detrimental to the body; some metabolites provide energy for cells, whereas others are toxic and must be removed from the body, for example viaurine. Professor David Wishart at the University of Alberta has been involved in the discovery and cataloguing of thousands of these metabolites for the past 15 years.

\section{THE DARK METABOLOME} Despite their importance in the dayscientay functioning of the human body of metabolites at present with the remaining $98 \%$ being referred to as the 'dark matter' of the metabolism, or the 'dark metabolome'. Although it was previously thought that scientists had a thorough understanding of metabolic processes, improvements in analytical chemistry technologies have led to the detection of many new, unknown metabolites. These unknown compounds are frequently detected using todays scientific instruments but cannot readily be identified and therefore limt scientists' current knowledge of human metabolism. It is this dark metabolome that Professor Wishart seeks to shime some light upon. Identifying these The BioTransformer is a free chemical prediction tool, developed to explore the mysterious dark metablome. increase our understanding of human

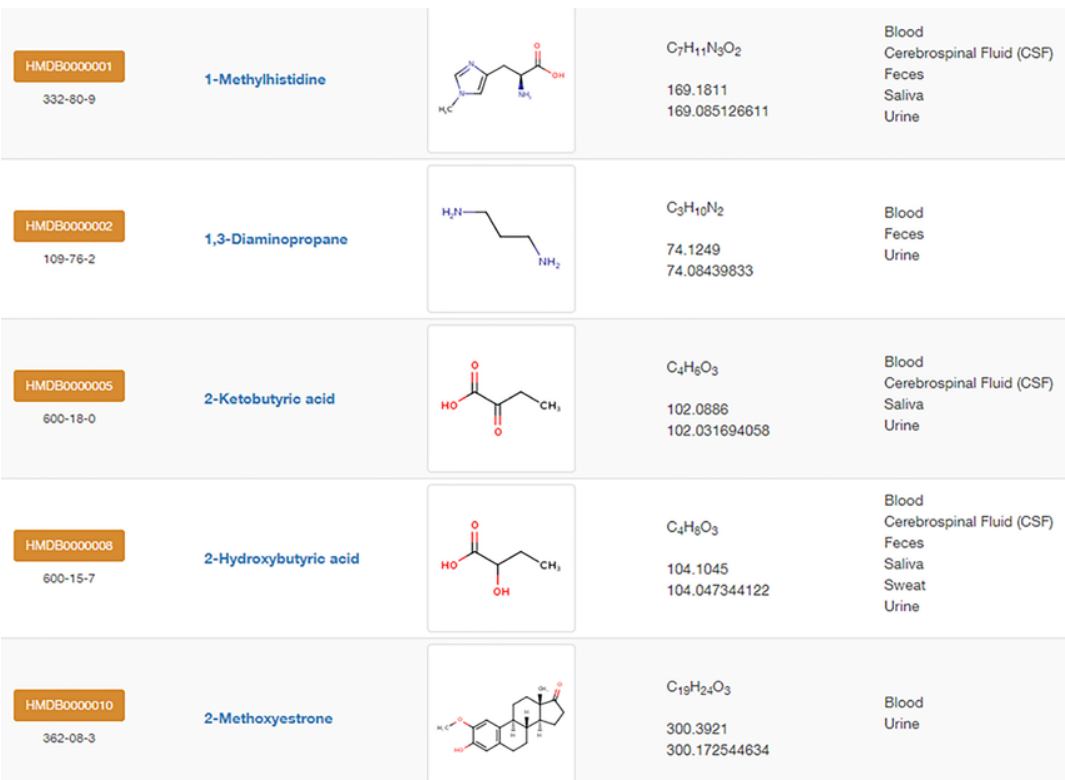

Identifying previously unknown metabolites will increase understanding of human physiology, disease and biochemistry.

physiology, disease and biochemistry. Furthermore, including the soil and aquatic microbiome in tracking these metabolic biotransformations will shed ight on the complex interactions and the metabolic 'life cycle' between humans and the environment, for example the impact of pesticides on human health.

Although much effort has gone into investigating the metabolic process involved in the production and a fats and proteins, less work hites, such on non-essential metabolites. These are the chemicals that are thought to make up much of the dark metabolome, and which are more often not derived from catabolism or anabolism, but from the biotransformation of other substances called xenobiotics. Xenobiotics are foreign substances such as drugs, plant chemicals, food additives or pesticides. There are hundreds of thousands of xenobiotics - and potentially millions of xenobiotic metabolites.

\section{USING MACHINES}

TO REPLACE MAN

One way that unknown metabolites

an be identified is through the use than would otherwise be possible. Isolating, purifying and determining the can take a skilled chemist one to two years. Using an in silico approach, this process can take a matter of minutes. If the dark metabolome consists of one million compounds (as currently thought), scientists predict it will take hundreds of years to characterise it manually. A faster, computational approach based on metabolism prediction appears to be the only option.

Metabolism prediction programmes typically start with a parent molecule and employ pattern recognition techniques, hand-made rules, or machine learning

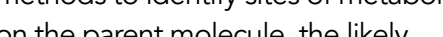
on the parent molecule, the likely rabolc reactions, and the chemical that site. Although a number of tools for metabolism prediction have already been developed, these were mainly designed to aid pharmacology-focused studies. As a result, today's metabolism prediction tools are not really suited for shedding light on the dark metabolome.

WHAT IS BIOTRANSFORMER? Professor Wishart and his team have used cutting-edge machine learning technology to develop a freely available software package that is specifically designed to help reveal the dark

The function of BioTransformer is two-fold; to predict small molecule metabolism using a metabolism prediction tool (BMPT), and to characterise unknown metabolites using BioTransformer predicts small mite. metabolism in mammals, as well as the 


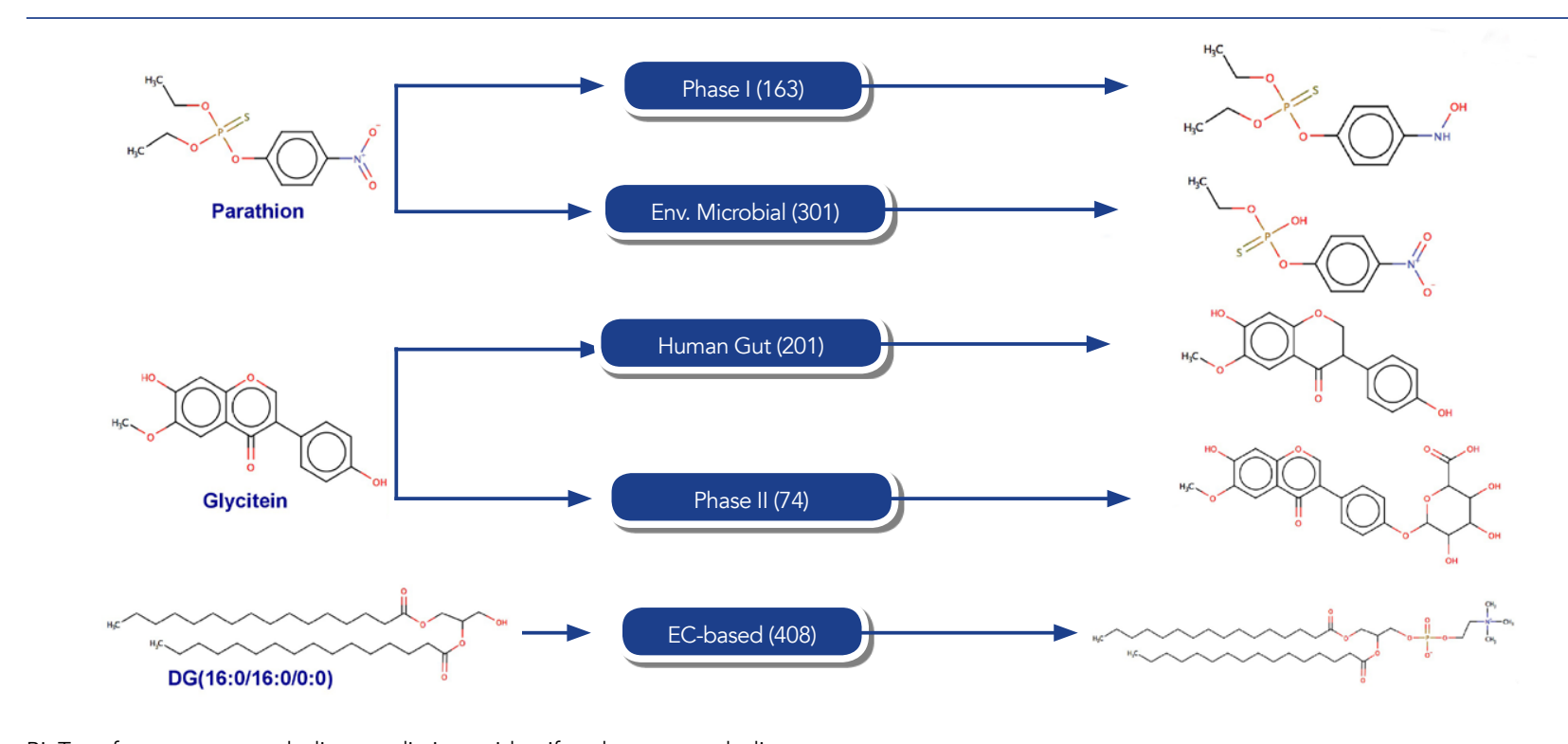

BioTransformer uses metabolism prediction to identify unknoun metabolites

gut microbiome, soil microbiome and

aquatic microbiome. More relevant to
mining the dark metabolome is the ability
which can be selected or combined

prediction to assist scientists in the

identification of unknown metabolites.

BioTransformer harnesses existing knowledge about known enzymatic reactions and uses pattern recognition and machine learning techniques almost any known chemical, including xenobiotics. The team at the University of Alberta developed and encoded almost 800 biotransformation rules, o 1,700 enzyme-based reaction rules, and multiple metabolic pathways in

(1)

(n) engine was incorporated to implement reaction site prediction and selection, as well as chemical product prediction. BioTransformer uses decision trees for its reasoning engine. Decision possible consequences, similar to the yes/no quizzes often found in magazines, and can select the most likely biotransformations or pathways for a particular situation. together by the user to meet specific based, CYP450 (or Phase I). Phase II. human gut microbial, and environmen (soil or aquatic) microbial. Each of of metabolic reactions, for example. CYP450 enzymes principally work in the liver and are responsible for first pass (Phase I) metabolism of many substances, including drugs; while Phase II reactions further transform, or lead to excretion of metabolites resulting from initial CYP450 metabolism.

To be useful for analysing the dark metabolome, BioTransformer had to bevent hroughly tested and metabolic predictions for a wide range of xenobiotics and endogen metabolites Indeed, Professor Wishart available tools, with results up to three times better than existing programmes.

Computational simulation of the process of human metabolism by BioTransformer allows scientists to rapidly

Professor Wishart describes

BioTransformer as a 'rocket ship' to help biochemists explore the unknown chemical universe within the human body. Prem the original chemicats. understanding of the dark metabolome. of BioTransformer to use metabolism to accurately predict the metabolism of trees model decisions and their these modules refers to a different has shown that BioTransformer is capable of outperforming other commercially predict hundreds of thousands of novel These computationally predicted, biologically feasible metabolites are now being used by Professor Wishart and his of the dark metabolome. In fact they have already used BioTransformer and mass spectrometry techniques to help identify a dozen previously identified xenobiotic (plant) metabolites in urine.

THE ROCKET SHIP

OF METABOLOMICS

Professor Wishart describes

Bio Transformer as a 'rocket ship'

that enables biochemists to explore the unknown chemical universe within the human body. One major benefit and frely avail hate, is open access expensive licensing arangemats not present bariers to research. This will encourage more researchers to use the tool and will foster an online, ideasharing community that can progress

Potential applications of advancing the capabilities of in silico metabolism prediction software include expansion of chemical databases of drugs, food compounds, environmental and environmentally relevant chemicals. Indeed, BioTransformer has the potentia to discover new biomarkers, bioactive metabolites and to inform development of drugs and household products, as well as facilitating the contaminants and other biologically

\section{Behind the Research}

\section{Professor David Wishart}

E: dwishart@ualberta.ca T: +1780-492-0383 W: http://biotransformer.ca/ W: http://www.wishartlab.com/

\section{Research Objectives} molecule metabolism in mammals, their gut microbiota, as well as the soil/aquatic microbiota. BioTransformer understand new aspects of metabolism.

\section{Detail}

Dept. of Biological Sciences

CW-405 Biological Sciences Bldg

University of Alberta

Edmonton, Alberta

Canada

Bio

Dr David Wishart is a Distinguished University Professor at the University of Alberta. For the past 12 years, he has led the "Human Metabolome Project," which is cataloguing all known metabolites in human tissues and biofluids. Prof Wishart is a member of The Metabolomics Innovation Centre (TMIC), Canada's national metabolomics laboratory.

\section{Funding}

- Genome Canada

\section{Collaborators}

- Claudine Manach (INRA - Clermont-Ferrand) - Russ Greiner (University of Alberta)

- Yannick Djoumbou-Feunang (University of Alberta)

- Jarlei Fiamoncini (INRA - Clermont Ferrand)

- Alberto Gil de la Fuente (CEU - Madrid)
BioTransformer is a software tool that predicts small

- The Canadian Institutes of Health Research

\section{References} Djoumbou-Feunang, Y., Fiamoncini, J., Gil-de-la-Feunte, A.,
Greiner, R., Manach, C. \& Wishart, D.S. (2019). BioTransformer a comprehensive computational tool for small molecule metabolism prediction and metabolite identification. $J$
Cheminform. $11(2)$. doi.org/10.1186/s13321-018-0324-5.

\section{Personal Response}

How much work does it take to keep BioTransform up to date?

We are dedicating two full-time staff members to keep BioTransformer up to date and to continue to improve its accuracy, speed and performance. It's proven to be much
more popular and more useful than we expected.

As with other software, will upgrades be required in the future?

II Certainly, as we learn more about metabolism and completely unexpected or unpredicted, we will have to upgrade and improve BioTransformer. It will always be evolving - at least until we've solved the problem of the dark metabolome.

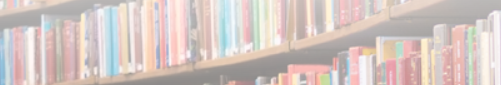

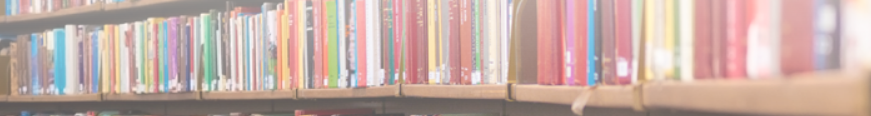

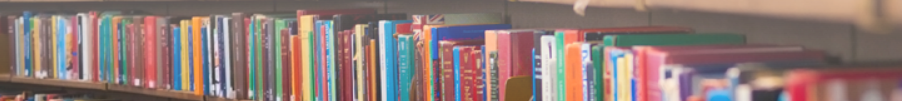

IIIIIIII 